\title{
Improving reconciliation following medical injury: a qualitative study of responses to patient safety incidents in New Zealand
}

\author{
Jennifer Moore, ${ }^{1}$ Michelle M Mello ${ }^{2}$
}

\begin{abstract}
${ }^{1}$ Faculty of Law, University of New South Wales, Sydney, New South Wales, Australia

${ }^{2}$ Stanford Law School, Stanford University, Stanford, California, USA
\end{abstract}

\section{Correspondence to} Dr Jennifer Moore, Faculty of Law, University of New South Wales, The Law Building, Sydney, NSW 2052, Australia; jennifer.moore@unsw.edu.au

Received 10 June 2016 Revised 13 February 2017 Accepted 15 February 2017 Published Online First 9 March 2017

\section{SLinked}

http://dx.doi.org/10.1136/ bmjqs-2017-006914

CrossMark

To cite: Moore J, Mello MM BMJ Qual Saf

2017;26:788-798.

\begin{abstract}
Background Despite the investment in exploring patient-centred alternatives to medical malpractice in New Zealand (NZ), the UK and the USA, patients' experiences with these processes are not well understood. We sought to explore factors that facilitate and impede reconciliation following patient safety incidents and identify recommendations for strengthening institutionled alternatives to malpractice litigation.
\end{abstract}

Methods We conducted semistructured interviews with 62 patients injured by healthcare in NZ, administrators of 12 public hospitals, 5 lawyers specialising in Accident Compensation Corporation (ACC) claims and 3 ACC staff. NZ was chosen as the research site because it has replaced medical malpractice litigation with a no-fault scheme. Thematic analysis was used to identify key themes from interview transcripts.

Results Interview responses converged on five elements of the reconciliation process that were important: (1) ask, rather than assume, what patients and families need from the process and recognise that, for many patients, being heard is important and should occur early in the reconciliation process; (2) support timely, sincere, culturally appropriate and meaningful apologies, avoiding forced or tokenistic quasi-apologies; (3) choose words that promote reconciliation; (4) include the people who patients want involved in the reconciliation discussion, including practitioners involved in the harm event; and (5) engage the support of lawyers and patient relations staff as appropriate.

Discussion Policymakers and healthcare institutions are keenly interested in non-litigation approaches to resolving malpractice incidents. Interviewing participants involved in patient safety incident reconciliation processes suggests that healthcare institutions should not view apology as a substitute for other remedial actions; use flexible guidelines that distil best-practice principles, ensuring that steps are not missed, while not prescribing a 'one size fits all' communication approach.

\section{INTRODUCTION}

Traditionally, litigation has provided the primary venue through which the stories behind patient safety incidents are aired. The shortcomings of this approach have led healthcare institutions to develop alternatives, such as communicationand-resolution-programmes (CRPs), to meet patients' needs. ${ }^{1}$ Yet little is known about the range of patients' needs following patient safety incidents, and thus about how well healthcare institutions meet them. ${ }^{2-4}$

Researchers have noted the challenges of undertaking work in this field. One group identified providers' 'reluctance to reveal the details of incidents and disclosures' and the 'protracted and confidential nature of incident investigations, complaints processes, and litigation proceedings' as barriers. ${ }^{2}$ Another challenge is gaining access to injured patients and family members, who institutions worry may be provoked to sue if researchers ask about their experiences.

Existing literature about injured patients' needs establishes some fundamentals: patients and families ${ }^{5}$ value efforts to prevent recurrences ${ }^{3} 6$ and disclosure communications that are honest, transparent, efficient and compassionate. $^{3}$ 7-9 Their accounts reveal that patient safety incidents often involve immense emotional, physical and financial impacts, ${ }^{10}$ which can be exacerbated by poor responses from providers. ${ }^{11} 12$

Yet many questions remain, particularly when one attempts to apply these 
findings to the context of hospital-based reconciliation programmes such as CRPs. Existing findings lack the granularity necessary to identify specific improvements to reconciliation processes that would better meet patients' needs. Given the paucity of information, it is perhaps unsurprising that providers' responses to patient safety incidents often fail to meet patients' expectations. ${ }^{4}{ }^{13}$ Studies have found a gap between patients' and providers' perspectives on disclosures $^{13} 14$ and identified barriers to effective disclosure, including clinicians' liability concerns. ${ }^{12} 1315$

The knowledge gap complicates health systems' efforts to improve institutional responses to patient safety incidents. Most disclosure policies adopted by governments, professional organisations and healthcare organisations suggest that providers offer an explanation of the incident, an apology, and a commitment to preventing recurrences. ${ }^{16-22}$ Guidance as to how to deliver these elements is in short supply, however, with some consensus documents stating that they do 'not attempt to prescribe...practices'. ${ }^{19}$ Beyond disclosure policies, efforts to implement CRPs are hampered by the absence of empirical information about how effective they are in meeting patients' needs. ${ }^{23-25}$

To identify factors that facilitate and impede reconciliation and recommendations for strengthening institution-led alternatives to litigation, we explored the experiences of injured patients and other stakeholders in New Zealand (NZ) using key informant interviews. NZ was chosen as the research site because it replaced malpractice litigation with the Accident Compensation Corporation (ACC), an administrative, no-fault compensation system. Our assumption was that NZ's medicolegal environment would be conducive to robust reconciliation processes, with lessons for more adversarial settings such as the USA and UK.

We define 'reconciliation' as engaging with patients and families about an unexpected care outcome and offering remediation. The goals of reconciliation are to acknowledge and, where possible, redress emotional, physical and financial harm; express an ethic of continuing care for the patient; and re-establish trusting relationships. We use 'reconciliation', rather than the more common 'resolution', to signal that the process goes beyond legal settlement and may not result in the incident being completely 'resolved' from the patient's perspective. In discussing medical injuries, we use the WHO's preferred phrase, 'patient safety incident', ${ }^{26}$ unless we are discussing the NZ context, in which case we refer to 'treatment injury' because that is NZ's statutory language.

Our project was undertaken at a time when policymakers and healthcare institutions in many countries are keenly interested in the effectiveness of nonlitigation approaches to resolving malpractice incidents. In 2016, the Agency for Healthcare Research and Quality released a toolkit to support nationwide implementation of the communication-and-resolution approach. ${ }^{27}$ Our findings point to specific practices that should be pursued in these programmes and in reconciliation processes in hospitals globally.

\section{METHODS}

\section{Setting}

In NZ, an island nation of 4.6 million, the government provides and finances healthcare services. ${ }^{28}$ Functioning alongside NZ's healthcare system is the ACC, a government-operated, tax-funded entity which is accountable to a parliamentary minister. The ACC scheme, which came into effect in 1974, ${ }^{29}$ extinguished New Zealanders' right to bring civil claims for personal injury damages. Instead, injured persons can apply to ACC for compensation and rehabilitation. ${ }^{30-32}$

\section{Recruitment of participants}

Because injured patients are a difficult population to reach, ${ }^{2}$ two approaches were used to recruit patients. First, we invited participation from members of Acclaim Otago, a national support group for injured people that is open to all ACC claimants, ${ }^{33}$ Study information was emailed to members who had filed treatment injury claims. Second, we used snowball sampling. ${ }^{34} 35$ Individuals were eligible for inclusion if they (1) were English-speaking patients or family members of patients who had experienced treatment injury, (2) had experienced a disclosure programme in a healthcare institution and (3) had made a claim to ACC. Forty-four participants were recruited through Acclaim and 18 were recruited using snowball sampling.

Hospital administrators were recruited by sending invitation letters to NZ's 20 public hospitals, known as District Health Boards (DHBs). We selected DHBs because (1) public hospitals comprise the majority of hospitals in NZ; (2) DHBs are among the provider groups involved in the greatest proportion of ACC claims and; (3) DHBs operate disclosure programmes. Within each DHB we invited senior managers whose role involved working with ACC and Chief Medical Officers to participate.

We recruited ACC staff by sending a recruitment letter to a senior individual from the Treatment Injury Center (TIC) at the ACC, the office that makes decisions on claims involving treatment injuries and collects data from these claims. He recommended that we also interview two of his colleagues. We included ACC staff in our sample because they have handled large numbers of treatment injury cases, interacting directly with claimants. Their insights shed further light on what patients tend to need after patient safety incidents and what tends to be helpful in fostering reconciliation.

We emailed recruitment letters to eight of NZ's practicing plaintiff lawyers who specialise in ACC 
claims. The recruitment packet included information about the project and researchers.

\section{Interview methods}

Interviews were semistructured and used an interview guide informed by our literature review. Interview questions for patients explored their experiences of treatment injuries and the institution's and ACC's response, factors that facilitated reconciliation, and suggestions for improving responses to treatment injuries. Questions for hospital administrators addressed hospitals' relationships with patients following treatment injuries, their experiences of incident response and treatment injury claims processes, and suggestions for improvement. Questions for ACC staff and lawyers covered their experiences with treatment injury claims, lawyers' and health providers' roles during claims, and reform options.

One investigator (JM) conducted the interviews between March and July 2015. Interviews with DHBs, lawyers and ACC TIC lasted 60-90 min. Interviews with injured patients or family members lasted 60-180 min, at the participant's discretion. Twenty-two participants chose telephone interviews and 60 chose face-to-face interviews, which were conducted at the participant's home or workplace. Each person was interviewed alone, except in two cases, where the patients opted to be interviewed with their wives. Interviews were audio recorded and transcribed verbatim.

\section{Data analysis}

Data were managed using NVivo V.11 software. Our thematic analysis ${ }^{36}$ of the interview data was undertaken by JM using a progressive process of classifying, comparing and refining text to create categories or themes. ${ }^{37}$ To ensure reliability, MM crosschecked the categories. Discrepancies were discussed and an agreeable interpretation was reached. Patterns of commonality and cases that 'didn't fit' were noted. ${ }^{38}$ For patients and family members, the interviews and the analysis were guided by the categories until data saturation was reached. For some questions, response frequencies were also recorded.

\section{RESULTS}

\section{Participant characteristics}

The 82 participants consisted of 56 patients and 6 family members, 3 ACC staff, 12 DHB administrators and 5 lawyers (table 1). The six family member participants were parents of injured children. Of the 103 treatment injury Acclaim members invited to participate, 62 agreed. Of the 20 DHBs invited to participate, 12 agreed. Of eight lawyers invited to participate, five agreed.

Table 2 describes the clinical areas in which the participants' treatment injuries occurred and table 3 the ACC's classifications of the severity of the
Table 1 Interview respondent demographics $(n=82)$

\begin{tabular}{|c|c|c|}
\hline & $\begin{array}{l}\text { Patients and family } \\
\text { members }(n=62)\end{array}$ & $\begin{array}{l}\text { Others } \\
(\mathrm{n}=20)\end{array}$ \\
\hline \multicolumn{3}{|l|}{ Age group (years) } \\
\hline 20-39 & 6 & 2 \\
\hline $40-64$ & 54 & 18 \\
\hline $65+$ & 2 & 0 \\
\hline \multicolumn{3}{|l|}{ Gender } \\
\hline Female & 35 & 10 \\
\hline Male & 27 & 10 \\
\hline \multicolumn{3}{|l|}{ Race/ethnicity } \\
\hline European & 38 & 18 \\
\hline Maori & 9 & 1 \\
\hline Pacific Islander & 8 & 0 \\
\hline Asian & 5 & 1 \\
\hline Other & 2 & 0 \\
\hline \multicolumn{3}{|l|}{ Education level } \\
\hline High school & 28 & 0 \\
\hline Polytechnic/trade school & 13 & 0 \\
\hline College & 17 & 17 \\
\hline Graduate school & 4 & 3 \\
\hline \multicolumn{3}{|l|}{ Years since initial injury* } \\
\hline$\leq 1$ & 4 & \\
\hline $2-3$ & 31 & \\
\hline $4-6$ & 13 & \\
\hline $7-9$ & 4 & \\
\hline $10-13$ & 6 & \\
\hline $14+$ & 4 & \\
\hline \multicolumn{3}{|l|}{ Professional role } \\
\hline Hospital administrator & & 12 \\
\hline ACC lawyer & & 5 \\
\hline ACC staff & & 3 \\
\hline
\end{tabular}

*Thirteen participants also experienced 'consequential injuries', which are defined in the ACC legislation as personal injuries that are 'a consequence of treatment given to the person for another personal injury for which the person has cover' (s 20(2)(d)), and personal injuries 'caused by a gradual process, disease, or infection consequential on treatment given to the person for personal injury for which the person has cover' (s 20(2)(h)). ACC, Accident Compensation Corporation.

participants' injuries. Nineteen per cent of patients experienced what would have been classified as 'medical error' (negligence) under the pre-2005 ACC legislation. ${ }^{39}$

\section{Interview themes}

Interviews revealed several important elements of the reconciliation process.

Ask

Almost all patients and lawyers commented that healthcare providers should elicit the patient's needs by asking, then listening carefully. As a lawyer explained, there 'has to be a window of vulnerability on the part of the hospital and doctor, where they relinquish control, and ask the patient which remedy they want' (participant 4, lawyer). All patients who 
Table 2 Clinical areas in which participants' injuries occurred

\begin{tabular}{ll}
\hline Clinical area & Number of participants \\
\hline Orthopaedics & 18 \\
Obstetrics and gynaecology & 13 \\
Internal medicine & 9 \\
Cardiology & 8 \\
General surgery & 3 \\
Paediatrics & 3 \\
Neurosurgery & 3 \\
Vascular surgery & 2 \\
Anaesthesiology & 1 \\
Oncology & 1 \\
Colon and rectal surgery & 1 \\
\hline
\end{tabular}

Table 3 Accident Compensation Corporation (ACC) treatment injury report severity classifications

\begin{tabular}{ll}
\hline ACC severity classification & $\begin{array}{l}\text { Number } \\
\text { of patients }\end{array}$ \\
\hline $\begin{array}{l}\text { Sentinel injury } \\
\text { (event resulted in death or major permanent loss of } \\
\text { function) }\end{array}$ & 23 \\
$\begin{array}{l}\text { Serious injury } \\
\text { (potential to result in death or major permanent loss of } \\
\text { function) }\end{array}$ & 25 \\
$\begin{array}{l}\text { Major injury } \\
\text { (event which results in short-term to medium-term }\end{array}$ & 8 \\
lessening of bodily function) & \\
$\begin{array}{l}\text { Minor injury } \\
\text { (event which results in minimal lessening of bodily } \\
\text { function) }\end{array}$ & 6 \\
\hline
\end{tabular}

reported positive feelings about the disclosure meetings stated that the healthcare providers 'asked [them] what they could do to help', instead of 'telling me what they thought I needed' (participant 14, patient).

A key reason that patients appreciated providers asking was because some patients' needs do not fit neatly into the expected categories of apology, compensation and learning. For example, after the preventable death of their baby, a couple wanted a dedication with their baby's name placed on the wall in the hospital. They stated, "we don't want our baby forgotten" and "we want our baby's life to have meaning. The plaque will serve as a reminder to staff to never let other parents endure the same horror" (participant 60, patient).

All patients reported that it is extremely helpful for the provider's response to be tailored to the needs and losses that patients have just expressed. Patients did not appreciate providers who 'repeated the script, even if it's not relevant to what [the patient] said' (participant 37, patient).

\section{Being heard}

There was widespread agreement among participants (73/82) that careful listening was difficult for providers and did not happen frequently. Only 17/62 (27\%) patients reported that they felt heard; 73\% said they did not. One patient commented (participant, 46, patient):

It's absolutely, fundamentally, about being heard and being able to look the health professionals in their eyes, tell your story, and for them to look you in the eyes, and actually register.

Patients who felt unheard complained that their provider 'constantly interrupted' (participant 2, patient). Several reported frustration that instead of listening to their narrative during meetings, providers focused on the information in the medical record and information they 'deemed clinically relevant' (participant 29, patient). In contrast, positive experiences were reported when efforts were made to listen to what patients had to say. An ACC manager stated (participant 22, ACC staff):

Patients who have suffered treatment injury do want to know that we have listened to their experiences...The idea that apology is all that matters, I don't think that is true for all patients...It takes a lot of time to listen properly, but you'll save a lot of time if you do that early on.

Participants emphasised that it is important to listen because for some, reconciliation can only be achieved if they feel the provider understands and validates their individual experience of loss. An interviewee from ACC noted that the claims process doesn't provide for claimants' 'emotional needs', leaving them 'feeling that they have not been heard' or 'given an opportunity to tell their story about the loss that they think has occurred and what that has meant to them' (participant 25, ACC staff).

Lawyers observed that when patients do not feel they have been heard (participant 19, lawyer):

It intensifies the client's grievances... .... of often hear clients say, 'This is the first time someone has listened to me'....When the 'being heard' element is missing, the compensation [ACC] and accountability [HDC, Health Practitioners' Disciplinary Tribunal] arms are often not enough for many patients.

Another noted, "When they didn't get the acknowledgement, admission, and being heard, my clients would go on a crusade" (participant 10, lawyer).

In contrast, the following patient's experience illustrates how listening can ease the path towards reconciliation (participant 55, patient):

What was really important was being able to tell them about what I had lost because of the injury. ...The treatment injury just destroys everything. My husband is afraid to touch me now in case he hurts me.... It's not just the big things, but the little everyday things like cleaning your house.... I got to tell [the hospital staff] about that and they listened to it all and didn't say that these things were little. It was that, not the compensation, that made me feel healed. It restored my trust in my doctor. 
Apology

Patients offered six insights about apology. First, while patients often appreciated apologies from staff who were not involved in the incident, patients $(59 / 62)$ were particularly glad to receive face-to-face apologies from the practitioner(s) involved in the incident (participant 51, patient):

There were lots of silences. But they were helpful spaces where I was given time to process what was said... I could see in [the doctor's] eyes that he was genuinely remorseful about the loss of my baby...that he really meant it when he apologized. I went into the meeting devastated. I came out feeling like I could move on. ...[W] actually eventually had another child and she was delivered at the same hospital.

Expressions of remorse from those uninvolved in the patient's care were deemed inauthentic. According to a patient (participant 6 , patient):

What I didn't appreciate, though, was when the manager kept taking over. ... The whole conversation came across as fake then. I wanted to hear what the doctor had to say, in his own words.

Second, when asked how, if at all, the timing of disclosure was relevant, all patients reported that apologies delivered months or years after the incident were not valued. One remarked, "An apology this late in the game means nothing. I actually didn't want one at this stage" (participant 5, patient). Yet, some patients $(21 / 62)$ reported that apologies are better received when the patient has had time to process the loss. For example, a mother reported (participant 60, patient):

The meeting was about 4 weeks after my baby passed. ... [That timing] worked for me. It was all too much to process in the beginning ... Information overload during grief.

Third, all patients reported that 'I'm sorry' may ring hollow when not accompanied by acknowledgement of the harm caused and how it has affected the patient's life, and an acceptance of responsibility. Apologies that were perceived as an item 'to put in a box and tick off' (participant 44, patient) were not valued. One patient commented that she "appreciated the apology from the surgeon because it wasn't just the simple 'I'm sorry'. He actually said he was sorry for the heart-wrenching ordeal I'd been through. I felt like he recognized how bad it had been for me" (participant 54, patient). For a number of patients $(14 / 62)$, it was important that the practitioner asked for forgiveness: 'The apology would have worked if the doctor said, "... Will you ever be able to forgive me for what I've done?" (participant 58, patient).

Fourth, for most patients $(52 / 62)$, apology is not a substitute for other forms of remediation, such as compensation. This sentiment seemed especially strong among seriously injured patients-for instance, one remarked that she 'lost my house because of his incompetent, botched surgery and had to move to a different city. An apology can't fix that' (participant 44, patient).

Nearly all patients $(59 / 62)$ underscored the importance of institutions making efforts to prevent recurrences. For instance, patients made comments such as 'an apology is worthless unless the hospital says exactly what they will do so that no one else goes through my horrendous experience' (participant 1, patient). Six of the 62 patients said that other forms of remediation were more important than apology. According to one, 'apologies are feel-good fluff' that 'don't do any good to change the system' (participant 9, patient). Patients felt aggrieved if they had to follow-up about patient safety efforts: 'It's not right that I should have to ask them what's happening to fix it. ... They should make it right and come to me to tell me what the results are' (participant 23, patient).

Patients and lawyers reported that it is helpful when institutions spend time ascertaining from patients and families whether an apology is sufficient or whether they are seeking other forms of redress. One patient explained: "I loved that [the hospital] asked me 'How can we address this for you?"”. (participant 37, patient). A lawyer also commented on the importance of 'open-ended offers', advising that providers ask (participant 8, lawyer),

\begin{abstract}
'What is your grievance? What can we do to remedy it? What do you want?' Let the patient say what the remedy is. But don't dictate it to them. Don't say 'I'm sorry', assuming that's what they want and then think that's it and you can all move on. I can say I have seen many clients grossly offended by that.
\end{abstract}

Compensation was frequently mentioned as an important complement to apology. One lawyer recounted (participant 11 , lawyer):

At the [mediation] the [hospital] said, 'We're sorry it happened. We'll give you counseling'. My client didn't want that. She said, 'No, I can't work. I can't pay the bills. I need some financial assistance to survive'.

Many of the indigenous New Zealander and Pacific Islander patients (13/17) articulated that for them, a 'culturally appropriate apology' included a money offer as a symbolic gesture (participant 18, patient):

The offender should offer money, but less as compensation, more as remorse. It's about restoring relationships.

Fifth, all participants noted that poorly executed apologies can do more harm than good. For example, one patient decided never to return to the hospital because he was so angered by the 'crap apology' in which he recalled the hospital stating, 'We're sorry you felt our care was disappointing' (participant 62, 
patient). Other patients perceived that the apologies they received were coerced and therefore inauthentic (participant 52, patient):

In that letter, [the physician] apologized, but it was crocodile tears. ...She was only apologizing then because she was being investigated.

Similarly, the lawyers described the problem of coerced apologies (participant 10, lawyer):

[The doctor] says 'sorry' at the appropriate, predetermined time, but only when jabbed by his boss, or the HDC. My experience is that clients do not appreciate those types of 'apologies'.

Finally, even though they are no panacea, apologies ordinarily should be offered, after the provider has listened carefully and elicited the individual patient's emotional needs.

Terminology and the need to choose words carefully

Many patients or family members (47/62), lawyers (5/ $5)$, hospital administrators (5/12) and ACC staff (3/3) spontaneously emphasised that providers' use of appropriate terminology could facilitate reconciliation, while poorly chosen words could undermine it. The most frequent and passionate comment from patients was that providers should not refer to the 'resolution' of the treatment injury because these events are never resolved from patients' perspective (participant 52, patient). Instead, patients preferred to say that they can achieve the 'ability to move on' if providers respond appropriately (participant 60 , patient):

Well, I suppose from their [the hospital's] perspective, it's 'resolved' because our discussions have ended, and our ACC claim was accepted. But it's not 'resolved' for us. Our baby is no longer with us and nothing will bring him back.

All patients also commented that clinicians should take care with their descriptions of injuries because patients' perceptions of the severity of the injury may differ from theirs. For example, one patient explained (participant 30, patient):

After the operation, [the nurse] said, almost flippantly, 'Oh well, at least your eyes aren't damaged, it's only your ears. Less harm done'. I am a musician. My ears are important!

\section{Right people in the room}

The primary insight offered by participants about the importance of involving the treating clinician/s in the process was that only the 'wrongdoer' can heal the emotional harm and restore the patient-practitioner relationship. As one patient explained: "I wanted mediation with the doctor who was responsible...I still can't get closure because I haven't yet spoken to that doctor" (participant 36, patient). All patients emphasised that the direct involvement of the treating provider was crucial to their emotional healing. For example, one patient explained (participant 31, patient):

There are four walls to your overall health-physical health, mental health, family, and relationships...If you only have one or two walls, the house will collapse...So, if a doctor hurts you, even if she didn't mean to, then the relationship wall has to be healed, just as much as her trying to physically heal you.

The lawyers indicated that in their experience, the patients who felt the treating practitioner/s failed to engage with them are the most likely to escalate their cases to appeal. Seven of the nine patients who were not given the opportunity to talk to the treating clinician(s) reported feeling aggrieved.

The role of lawyers and support persons

In NZ, patients can file claims free of charge through a healthcare provider, who need not be the clinician involved in the treatment injury. DHB administrators reported that they do not routinely involve their own lawyers in treatment injuries, although they often seek legal counsel if the case is complex.

Patients who sought assistance from lawyers valued their compassion and ability to facilitate space for them to be heard. One lawyer remarked, "A lot of what patients tell me, during what I call the 'crying chair sessions', is not legally material. But it is extremely material to the patient" (participant 19, lawyer). Four patients described their lawyers as 'angels' (participants 60, 62, 52, 44).

Because the lawyers were so helpful, patients appreciated practitioners or institutions who notified them that they could seek assistance from an advocate or lawyer. As one patient remarked, "I regret not getting a lawyer sooner...It was really helpful that the hospital said I could have help from a lawyer, because I hadn't thought of that and he really helped to explain stuff to me" (participant 44, patient).

All patients reported that they appreciated having a support person-whether a lawyer, family member or advocate-present during the initial disclosure discussion with the clinician. Many reported feeling too vulnerable and sick to process the information that the clinician offered.

\section{DISCUSSION}

Our findings generally accord with points that have been suggested in normative work on disclosure in the USA and in prominent disclosure guidelines. ${ }^{19}$ 40-42 They confirm the salience of elements in reconciliation (explanation, apology, compensation and learning) outlined in previous empirical research, but highlight the importance of an additional elementbeing heard. They suggest several areas in which US disclosure policies and CRP practices could evolve to better serve patients' needs (table 4). 
Table 4 Recommendations for reconciliation conversations emerging from key informant interviews

Question
Eliciting patients' and family members' needs
Conducting reconciliation conversations with emotional
intelligence

Recommendation

- Explicitly ask what patients'/families' needs are. Do not make assumptions—even that apology is desired.

- Recognise that although most patients want an explanation, apology, compensation and learning, for many patients, simply being heard is most important and must occur early in the disclosure process.

intelligence

- Time the conversation(s) appropriately.

- Although early contact (within 48 hours of the incident) is important, some patients need time to process the incident, necessitating multiple conversations.

- The onus is on institutions to follow-up with patients/families who need time to process the incident.

- In arranging follow-up communications, elicit what timing works best for patients/ families.

- Offer apologies in a manner that maximises their effectiveness in promoting reconciliation:

- Offered in person by the clinician(s) involved in the incident.

- Offered after the provider has listened carefully to the patient and elicited his/her needs. This may involve not including an apology or offering a culturally appropriate apology or an apology which seeks forgiveness.

- Timed according to the patient's needs, but generally not postponed until late in the process.

- Includes more than 'I'm sorry'; explicitly acknowledges the harm and the impact on the patient/family.

- Not offered as a substitute for other forms of remediation such as compensation and proof of learning.

- Executed skilfully and without coercion.

- Respect cultural differences.

- Reflect upon whether the process is culturally sensitive; ask questions as needed.

- Ask what a meaningful apology includes in the patient's cultural or religious tradition, if appropriate.

- Avoid insensitive word choices.

- Contemplate wording ahead of the conversation, recognising that it is important to patients and can help or harm the reconciliation process.

- Refer to 'patient safety incidents' instead of 'errors'.

- Replace 'resolution' with 'reconciliation'.

- Be open to patients' differing interpretations of what constitutes 'harm' and 'severity'.

Convening the right people to participate in reconciliation discussions

- Ask patients who they want involved.

- Include the clinician(s) involved in the harm event, unless the patient indicates this is undesirable.

- Include support people such as lawyers and patient relations staff and notify patients of their right to consult a lawyer.

Responding to patients and family members who are particularly concerned with preventing recurrences

- In disclosure conversations, share any patient safety lessons learned and actions taken. If the incident is still under investigation, assure patients/families that a review is being done.

- If learning takes place over a longer time frame, be proactive about recontacting patients/families to share the patient safety steps taken.

Improving reconciliation guidelines

- Strict protocols undermine the objective of tailoring institutional responses to individual patients'/families' needs.

- Use flexible guidelines that distil best-practice principles, ensuring that steps are not missed while not prescribing a 'one size fits all' communication approach.

- Where necessary, amend guidelines to reflect the above best practice principles. Add (or, if it is already present, emphasise) the 'being heard' element of the disclosure process.

- Move the being heard element to the first step in the initial and formal disclosure discussions.

- Add a step that requires asking patients open questions such as 'how can we help you?'

\section{How can institutions execute reconciliation processes with emotional intelligence?}

In addition to creating space for injured patients to be heard, institutions can promote reconciliation by being mindful of three elements: apology, culture and terminology.
Apology enjoys widespread endorsement as an essential element of successful reconciliation. $\begin{array}{llll}16 & 19 & 20 & 43-45\end{array}$ But apologies can easily fail, causing harm, ${ }^{46}$ especially when institutions include apology as an item on their disclosure checklist but are inattentive to its authenticity. Our study echoes previous research 
findings that apologies do not always lead to 'closure' because some patients perceive 'I'm sorry' as a 'Band Aid', devoid of 'accountability' or 'results'. ${ }^{3}$ Our findings lend empirical weight to philosophical arguments that coerced apologies may be harmful ${ }^{42} 4748$ and to Lazare's work highlighting the importance of the timing of apologies and who offers them. ${ }^{46} 49$ They are consistent with legal ${ }^{42}$ and empirical ${ }^{3}$ research suggesting that patients consider an apology more authentic if provided by the involved clinician(s).

Authentic apology-that is, genuine acceptance of responsibility, expression of regret and commitment to efforts to prevent recurrences-should be offered in the patient's 'language of apology.$^{50}$ One such language involves inviting the "party harmed to extend forgiveness and, thus, provide the opportunity for reconciliation'. ${ }^{42}$ Forgiveness is an especially important element for ethnic groups such as Maori and Pacific Islanders.

Previous research has asked how disclosures can be made 'culturally sensitive and relevant', ${ }^{2}$ yet the question remains largely unanswered. Our findings emphasise the importance of reflecting upon whether the patient may need a 'cultural apology'. ${ }^{51}$ US consensus statements lack guidance about how to ensure that processes are culturally sensitive.

Terminology is also important in disclosure conversations. The importance of a conceptual framework and preferred terminology for patient safety has been recognised recently, ${ }^{26}{ }^{52}$ and countries such as NZ, UK and Canada have changed their terminology, ${ }^{17} 18$ yet some US consensus statements do not provide recommendations about terminology that should be avoided or used. ${ }^{19} 2122$ Research in the USA suggests that providers do 'choose [their] words carefully', but may do so in ways that obscure the fact of error or their responsibility for it, rather than in ways that respond to patients' needs. ${ }^{53}$

Although the label 'communication-and-resolution programs' is now in common use in the USA, ${ }^{54}$ our study suggests that institutions should consider replacing 'resolution' with an alternative like 'reconciliation'. 'Resolution' implies that the goal is to enable the institution to consider the case closed, not necessarily to heal relationships. Taft has written extensively about the limitations of 'resolution without reconciliation'. ${ }^{42} 48$

Institutions should also reflect upon what constitutes 'harm', their severity classifications and how these are explained to patients. Patients' and clinicians' definitions of 'harm' and 'severity' often differ. ${ }^{55}$ Medical sociologists and ethicists have argued that defining harm is value-laden and called for a patient-centred framing of 'harm'. ${ }^{56}$ Our findings lend weight to this call.

\section{Who should be involved?}

Institutions should ask injured patients who they would like involved in meetings about the patient safety incident; ordinarily, this will include the treating provider(s) and a support person. In contrast, some American consensus statements on disclosure neither indicate who should be involved ${ }^{21}$ nor recommend that patients be asked. ${ }^{21} 22$

Our conclusion is consistent with other evidence that patients want the clinicians involved in the event to understand what went wrong and be involved in disclosure discussions. ${ }^{3}{ }^{4}$ Some institutions may find the prospect of routinely involving treating providers challenging: what if the clinician in question is 'not a people person', as one respondent put it, or not to blame for the incident?

Our respondents expressed a clear preference for speaking with the involved provider even under these circumstances. Institutions can coach clinicians through these difficult conversations-and warn patients when a provider may struggle through them -but should not deny patients this critical aspect of reconciliation. Further, clinicians should understand that the goal of talking with the patient is not always to accept blame. Most patient safety incidents are not caused by error, and most adverse events are largely attributable to 'systems failures', not errors by individual providers. In such circumstances, the goal of communication is to achieve a mutual understanding of what occurred and reconciliation.

With regard to support persons (eg, lawyers, family members), our findings echo conclusions from Australian $^{11}$ and Canadian ${ }^{58}$ studies that patients often feel unsupported after patient safety incidents and therefore can benefit immensely from support persons. Because patients are often unaware of this option, ${ }^{3} 11$ institutions should routinely notify patients that they can involve a support person.

In the USA and UK, involving lawyers helps ensure that patients' interests are protected but could make the tenor of discussions more adversarial or provoke anxiety in clinicians. Experienced attorneys who approach reconciliation as a collaborative process are most likely to provide patients the support they need while facilitating timely, appropriate reconciliation. ${ }^{59}$ Recent US research has found that patients value their lawyers' roles in the process (Moore et al 2016, unpublished manuscript) and that attorney participation tended to help the process. ${ }^{1}$

\section{Should institutions use protocols?}

CRP architects have sought to introduce algorithms or 'key elements' for responding to patient safety incidents as a means of ensuring consistency of process. ${ }^{60}$ But risk managers sometimes argue that protocolising the process is misguided because each event is unique. $^{23}$ Are they right? Consistent with other research, ${ }^{5}$ the experiences of our participants do highlight the importance of tailoring institutional responses to individual patients' needs. That points 
away from using strict protocols-but does not mean guidelines should be abandoned.

Guidelines for communication and reconciliation could be strengthened by making 'being heard' a prominent step in the process. Legal therapeutic jurisprudence literature recognises the 'therapeutic value of telling one's story and being heard and of a procedure that values the ongoing, continuous relationship between the parties'. ${ }^{61}{ }^{62}$ Ensuring that patients are heard can involve asking open questions such as, 'How can I help you?' ${ }^{63}$ and listening carefully to answers. Asking this question and others is important because patients do not have homogeneous needs, and not all spontaneously express their concerns. Providers should be alert for needs that transcend the expected categories of apology, compensation and learning. ${ }^{64}$ They should also anticipate that the process may require multiple conversations, though some consensus statements contemplate a single discussion. 22

Some, ${ }^{19}{ }^{21}$ but not all, US consensus statements on disclosure recommend that providers engage in 'active listening'. In our study and others, ${ }^{366}$ injured patients frequently expressed the perception that their providers had not listened. Thus, attempts at 'listening' do not always give rise to feelings of 'being heard'. Being heard requires asking open-ended questions and allowing patients' priorities to lead the conversation.

\section{Study strengths and limitations}

Five strengths of this study were: (1) the participation of 56 patients and 6 family members; (2) the nuanced insights into the complexity of patients' responses generated by the qualitative method; (3) the gender and ethnic diversity of the sample; (4) the inclusion of multiple stakeholders; (5) the candour with which participants shared their views. Three limitations of this study were: (1) the recruitment methods may not have produced a nationally representative sample of all patients with treatment injuries; (2) there may be different elements for successful reconciliation that would be identified by another sample of participants; and (3) respondents may have consciously or unconsciously failed to mention other factors that influenced the extent to which they felt reconciliation had been achieved.

\section{CONCLUSION}

Patients' and families' experiences of reconciliation are central to assessing the success of these processes. Our participants' accounts underscore the importance of using flexible guidelines that do not prescribe a 'one size fits all' approach. As institutions with disclosure programmes make improvements, the insights from this study's participants should provide guidance and inspiration. Through listening to patients' stories about the impact of patient safety incidents on their lives, more patient-centred safer healthcare and reconciliation become possible.

Acknowledgements The authors thank the study participants for sharing their experiences; the reviewers; and Malcolm McDonald for thoughtful comments on earlier drafts of the manuscript.

Contributors JM and MMM developed the study idea and conducted the analyses. JM conducted the interviews and wrote the first draft of the manuscript. MMM helped revise the draft manuscript. Both authors reviewed and agreed on the submitted version of the manuscript.

Funding The Commonwealth Fund, a private independent foundation based in New York City.

Disclaimer The views presented here are those of the authors and not necessarily those of The Commonwealth Fund, its directors, officers or staff.

Competing interests None declared.

Ethics approval University of Otago Human Research Subjects Ethics Committee.

Provenance and peer review Not commissioned; externally peer reviewed.

Data sharing statement Following standard practice in this type of sensitive research with injured patients, we promised participants that their data would remain confidential. Specifically, the standardised information sheet we were required to distribute by the University of Otago's Human Subject Research Ethics Committee's states that only the researchers will have access to the data. Absent informed consent, we are not able to make the data broadly available. Should the reviewers or editors have questions about the validity of our data analysis or conclusions, however, we will of course do whatever we can to provide the information necessary to allay these concerns, short of violating our promises to participants.

\section{REFERENCES}

1 Mello MM, Boothman RC, McDonald T, et al. Communication-and-resolution programs: the challenges and lessons learned from six early adopters. Health Aff (Millwood) 2014;33:20-9.

2 Iedema R, Allen A, Britton K, et al. Patients' and family members' views on how clinicians enact and how they should enact incident disclosure: the "100 patient stories" qualitative study. BMJ 2011;343:d4423.

3 McVeety J, Keeping-Burke L, Harrison MB, et al. Patient and family member perspectives of encountering adverse events in health care: a systematic review. JBI Database System Rev Implement Rep 2014;12:315-73.

4 Mazor K, Greene S, Roblin D, et al. More than words: patients' views on apology and disclosure when things go wrong in cancer care. Patient Edu Couns 2013;90:341-6.

5 Mazor KM, Goff SL, Dodd KS, et al. Parents' perceptions of medical errors. J Patient Saf 2010;6:102-7.

6 Iedema R, Mallock N, Sorensen R, et al. Final report: evaluation of the National Open Disclosure Pilot Program. Sydney, The Australian Commission on Safety and Quality in Health Care. 2008.

7 Duclos CW, Eichler M, Taylor L, et al. Patient perspectives of patient-provider communication after adverse events. Int J Qual Health Care 2005;17:479-86.

8 Hovey RB, Dvorak ML, Burton T, et al. Patient safety: a consumer's perspective. Qual Health Res 2011;21:662-72.

9 Paterson R. The good doctor: what patients want. Auckland: Auckland University Press, 2012. 
10 Gallagher TH. A 62-year-old woman with skin cancer who experienced wrong-site surgery: review of medical error. JAMA 2009;302:669-77.

11 Iedema R, Sorensen R, Manias E, et al. Patients' and family members' experiences of open disclosure following adverse events. Int J Qual Health Care 2008;20:421-32.

12 O'Connor E, Coates H, Yardley I, et al. Disclosure of patient safety incidents: a comprehensive review. Int J Qual Health Care 2010;22:371-9.

13 Gallagher TH, Waterman AD, Ebers AG, et al. Patients' and Physicians' Attitudes Regarding the Disclosure of Medical Errors. JAMA 2003;289:1001-7.

14 Wu AW, Huang IC, Stokes S, et al. Disclosing medical errors to patients: it's not what you say, it's what they hear. J Gen Intern Med 2009;24:1012-17.

15 Manser T, Staender S. Aftermath of an adverse event: supporting health care professionals to meet patient expectations through open disclosure. Acta Anaesthesiol Scand $2005 ; 49: 728-34$.

16 New Zealand Health and Disability Commissioner. Guidance on open disclosure policies. Wellington: New Zealand Health and Disability Commissioner, 2007.

17 UK National Patient Safety Agency. Being open: communicating patient safety incidents with patients, families and carers. London: NHS, 2009.

18 Canadian Patient Safety Institute. Canadian disclosure guideline. Edmonton: Canadian Patient Safety Institute, 2008.

19 Massachusetts Coalition for the Prevention of Medical Errors. When things go wrong: responding to adverse events. (A consensus statement of the Harvard Hospitals, Boston). Boston: Massachusetts Coalition for the Prevention of Medical Errors, 2006.

20 Australian Commission on Safety and Quality in Health Care. Australian open disclosure framework: better communication, a better way to care. Sydney: Australian Commission on Safety and Quality in Health Care, 2013.

21 Gallagher TH. Training doctors to disclose unanticipated outcomes to patients: randomized trial. Agency for Health Care Research and Quality. 2010.

22 Collaborative for Accountability and Improvement. Communication and Resolution Programs: What are They and What do They Require? Seattle: Collaborative for Accountability and Improvement, 2015.

23 Mello MM, Senecal SK, Kuznetsov Y, et al. Implementing Hospital-Based Communication-And-Resolution Programs: Lessons Learned In New York City. Health Aff (Millwood) 2014;33:30-8.

24 Mello MM, Armstrong S, Gallagher TH. Can Communication-and-resolution Programs Succeed Where Multiple Organizations Must Collaborate? Working Paper. 2016.

25 Mello MM, Greenberg Y, Senecal SK, et al. Case Outcomes in a Communication-and-resolution Program in New York Hospitals. Working Paper. 2016.

26 Wu AW, McCay L, Levinson W, et al. Disclosing adverse events to patients: international norms and trends. J Patient Saf 2017;13:43-9.

27 Agency for Healthcare Research and Quality. Communication and optimal resolution (CANDOR) toolkit. Agency for Heathcare Research and Quality, 2016. http://www.ahrq.gov/ professionals/quality-patient-safety/patient-safety-resources/ resources/candor/introduction.html (accessed 11 Oct 2016).
28 Gauld R. Questions about New Zealand's health system in 2013, its 75th anniversary year. N Z Med J 2013;126:68-74.

29 Accident Compensation Act 1972.

30 (5 August 2004). 165 New Zealand Parliamentary Debates.

31 Accident Compensation Corporation and the Department of Labour. Review of ACC medical misadventure: consultation document. Wellington, 2003.

32 Accident Compensation Corporation. Overview of treatment injury July 2005 to June 2015. Wellington, 2015.

33 Acclaim Otago (Inc). A Support Group for Injured People and their Families. http://acclaimotago.org/ (accessed 8 Feb 2016).

34 Noy C. Sampling knowledge: the hermeneutics of snowball sampling in qualitative research. Int J Soc Res Methodol 2008;11:327-44.

35 Atkinson R, Flint J. Accessing hidden and hard-to-reach populations: snowball research strategies. Soc Res Update 2011;33:1-4.

36 Braun V, Clarke V. Using thematic analysis in psychology. Qual Res Psychol 2006;3:77-101.

37 Fossey E, Harvey C, McDermott F, et al. Understanding and evaluating qualitative research. Aust N Z J Psychiatry 2002;36:717-32.

38 Becker H. Tricks of the trade: how to think about research as you're doing it. Chicago and London: Chicago University Press, 1998.

39 Accident Compensation Corporation. ACC Background Paper: Overview of Accepted Medical Error Claims. Wellington: Accident Compensation Corporation, 2002.

40 McDonald TB, Helmchen LA, Smith KM, et al. Responding to patient safety incidents: the "seven pillars". Qual Saf Health Care 2010;19:e11.

41 Berlinger N. Avoiding cheap grace. Medical harm, patient safety, and the culture(s) of forgiveness. Hastings Cent Rep 2003;33:28-36.

42 Taft L. Apology and Medical Mistake: opportunity or foil? Ann Health Law 2005;14:55-94.

43 Wojcieszak D, Saxton J, Finkelstein M. Sorry works!: Disclosure, apology, and relationships prevent medical malpractice claims. Bloomington, IN: AuthorHouse, 2007.

44 Canadian Medical Protective Association. Communicating with your patient about harm: disclosure of adverse events. Ottawa: the Canadian Medical Protective Association, 2008.

45 National Patient Safety Agency. Being open: Saying sorry when things go wrong. London, 2009.

46 Lazare A. Apology in medical practice: an emerging clinical skill. JAMA 2006;296:1401-4.

47 McLennan S, Walker S, Rich LE. Should health care providers be forced to apologise after things go wrong? J Bioeth Inq 2014;11:431-5.

48 Taft L. Apology subverted: The commodification of apology. Yale Law J 2000;109:1135-60.

49 Lazare A. On Apology. Oxford: Oxford University Press, 2005.

50 Chapman G, Thomas J. The five languages of apology. Chicago: Northfield, 2008.

51 Lutui JB. Apology: a moral, cultural and restorative perspective. In: Therapeutic jurisprudence: New Zealand perspectives. Warren Brookbanks. (ed) Wellington: Thomson Reuters, 2015:71-91.

52 World Health Organization. More than words: conceptual framework for the international classification for patient safety. Geneva: WHO, 2009.

53 Gallagher TH, Garbutt JM, Waterman AD, et al. Choosing your words carefully: how physicians would disclose harmful 
medical errors to patients. Arch Intern Med 2006;166:1585-93.

54 Gostin L, Jacobson P, Studdert D, et al. Law and the health system. St. Paul, MN: Foundation Press, 2014.

55 Iedema R, Mallock N, Sorensen R, et al. The National Open Disclosure Pilot: evaluation of a policy implementation initiative. Med J Aust 2008;188:387-400.

56 Sharpe VA, Faden AI. Medical Harm: Historical, Conceptual and Ethical Dimensions of Iatrogenic Illness. Cambridge: Cambridge University Press, 2001.

57 Conrad P. The shifting engines of medicalization. J Health Soc Behav 2005;46:3-14.

58 Kovacs Burns K. Canadian patient safety champions: collaborating on improving patient safety. Healthc Q 2008;11:95-100.

59 Massachusetts Alliance for Communication, and Resolution following Medical Injury. Best Practices for Attorneys Representing Patients in Resolution of Medical Injury Using the CARe Approach. Massachusetts Alliance for Communication and Resolution following Medical Injury. http://www.macrmi.info/files/6614/5994/8225/Best_Practices for_Attys_Representing_Patients.pdf (accessed 9 Oct 2016).
60 Mello MM, Armstrong SJ, Greenberg Y, et al. Challenges of implementing a communication-and-resolution program where multiple organizations must cooperate. Health Serv Res 2016;51(Suppl 3):2550-68.

61 Acclaim Otago Inc. Understanding the problem: an inductive analysis of ACC appeals-access to justice for injured New Zealanders. Dunedin: Acclaim Otago, 2015.

62 Wexler DB. Therapeutic Jurisprudence in the Appellate Arena. Seattle Univ Law Rev 2000;24:217-22.

63 Berwick DM. What "patient-centered" should mean: confessions of an extremist. Health Aff (Millwood) 2009;28: w555-65.

64 Coletta SL. "Fix it as best you can, and learn from your mistakes": apology lessons, past and present. Front Health Serv Manage 2012;28:36-41.

65 Hall C. Ethics Consultant is a Master of Apologies. The Dallas Morning News. 2010. http://www.dallasnews.com/business/ columnists/cheryl-hall/20100612-Ethics-consultant-is-a-masterof-4304.ece (accessed 8 Oct 2016).

66 Villette M. For want of a four-cent pull chain. BMJ Qual Saf 2011;20:986-90. 